\title{
Anticancer drug cis-4-hydroxy-L-proline: Correlation of preclinical toxicology with clinical parameters of liver function
}

\author{
HEATHER DICKENS ${ }^{1}$, ANETT ULLRICH ${ }^{2}$, DIETER RUNGE $^{2}$, BERNO MUELLER $^{3}$, \\ ULRIKE OLSZEWSKI $^{4}$ and GERHARD HAMILTON ${ }^{4}$ \\ ${ }^{1}$ University of Leeds, Leeds, UK; ${ }^{2}$ PRIMACYT Cell Culture Technology, Schwerin; ${ }^{3}$ RIEMSER Arzneimittel, \\ Riems, Germany; ${ }^{4}$ Ludwig Boltzmann Cluster Translational Oncology, Vienna, Austria
}

Received January 31, 2008; Accepted March 13, 2008

\begin{abstract}
Hydroxy-L-proline (CHP) is being clinically evaluated as an anticancer drug. Since this compound targets the production of L-proline-rich proteins and critical L-proline residues, its impact on long-term cultures of human hepatocytes and toxicity in rats was studied to investigate possible effects on hepatic function, previously reported in rat hepatocytes. In the HEPAC ${ }^{2}$ human hepatocyte culture system, concentrations of CHP below $3.2 \mathrm{mg} / \mathrm{ml}$ had no significant effects on the release of lactate dehydrogenase (LDH), albumin, and urea. In rats, continuous administration of three different doses of CHP were tested for 28 days and resulted in signs of liver damage, as indicated by elevations of alanine aminotransferase (ALAT) and aspartate aminotransferase (ASAT) at a dose of $903 \mathrm{mg} / \mathrm{kg}$, corresponding to a plasma concentration of approximately $200 \mu \mathrm{g} / \mathrm{ml}$. Data from a clinical study of CHP in bladder and prostate cancer patients showed no adverse effects of administration of $8 \mathrm{~g} \mathrm{CHP/day,} 4$ days/week for 3 weeks in liver parameters ALAT, ASAT, $\gamma$-glutamyltransferase $(\gamma$-GT) and alkaline phosphatase (AP). In conclusion, the HEPAC ${ }^{2}$ human hepatocyte culture system correlates well with clinical results of a Phase II study of CHP, whereas a previous rat hepatocyte culture system predicted the compound would have toxic effects. The HEPAC ${ }^{2}$ system therefore constitutes a valuable tool for the preclinical screening of the
\end{abstract}

Correspondence to: Dr Gerhard Hamilton, Ludwig Boltzmann Cluster Translational Oncology, c/o Balderichgasse 26/13, A-1170 Vienna, Austria

E-mail: gerhard.hamilton@univie.ac.at

Abbreviations: AAP, acetaminophen; ALAT, alanine aminotransferase; AP, alkaline phosphatase; ASAT, aspartate aminotransferase; CHP, cis-4-hydroxy-L-proline (CHP); EGF, epidermal growth factor; $\gamma$-GT, $\gamma$-glutamyltransferase; HEPAC ${ }^{2}$, hepatocyte culture system; HGF hepatocyte growth factor; LDH, lactate dehydrogenase; MEM, minimal essential medium

Key words: liver, hepatocyte, cis-4-hydroxy-L-proline, toxicity, preclinical testing hepatotoxicity of chemotherapeutic and other drugs, thereby reducing the need for experimental animals.

\section{Introduction}

Toxicology studies in animals are required by regulatory authorities worldwide to provide assurance that the clinical testing of pharmaceutical drug candidates can be conducted safely. Safety concerns resulting from animal studies account for over $20 \%$ of retractions from drug development, while a retrospective study of animal tests compared to clinical experiences with 150 drugs revealed that only $43 \%$ of human toxicities had been correctly predicted (1). The number of compounds falsely rejected based on toxicity in animal models is unknown. Although hepatotoxicity is a frequent concern with all medications, chemotherapeutic agents are more commonly implicated in liver damage than most other drug classes (2). Cytotoxic therapy directed to rapidly growing cancer cells can easily impact hepatocytes, even though hepatocytes divide more slowly. Antineoplastic agents exerting hepatotoxic effects include common drugs, such as methotrexate, gemcitabine and taxanes, and newer drugs such as gefitinib and bortezomib, among others (3).

CHP has been characterized as an inhibitor of the collagen synthesis of fibroblasts. It is preferentially incorporated into L-proline-rich proteins, such as collagens, preventing the correct hydroxylation of the L-proline residues and the assembly of the triple helix. Most of the misfolded chains are degraded intracellularly. Therefore, CHP exerts antifibrotic effects and has additionally been shown to inhibit the proliferation of selected normal cell types as well as malignant cells. Pancreatic cancer and other tumor cells are damaged in response to treatment with $\mathrm{CHP}$ via induction of endoplasmatic reticulum stress and other mechanisms $(9,10)$. Following in vitro studies and a pilot clinical trial, CHP is currently under clinical development. Since CHP is incorporated into all proteins, critical L-proline residues may be modified in non-collagenous proteins and disturb cell functions leading to toxic side effects, for example in liver tissue, which is highly active in protein synthesis.

In vitro models can be considered highly advantageous in toxicology, not only due to the reduced necessity for animal use, but also because they allow for the use of human cell lines or tissues, thus creating results that are of increased relevance 
to toxicity and disease in humans $(11,12)$. In addition, an appropriate in vitro model saves time when evaluating new drug candidates and allows for the development of safer drugs. Primary hepatocyte cultures have been the predominant tool for in vitro cell culture hepatotoxicologic assessment (13). Hepatocytes have been shown to express most of the functions of the intact liver, and are therefore particularly suitable for biotransformation studies of xenobiotics (14).

Lee et al cultured isolated rat primary hepatocytes between two layers of collagen in a sandwich configuration that reinstates the cellular polarity necessary for long-term function in vitro (15). In this system, maintenance of hepatocyte function, as measured by the secretion of albumin, was shown to be dependent on both the sandwich gel configuration and the continued presence of L-proline in the culture media. CHP inhibited the response of sandwiched rat hepatocytes to L-proline in a dose-dependent and reversible manner. The addition of CHP to these cultures established for seven days also resulted in the inhibition of hepatocyte function.

Primary hepatocytes are an important model system for the analysis and study of liver-specific processes and functions. However, interspecies differences in all aspects of hepatocyte function exist and have been recognized and investigated for more than 30 years (11). Therefore, primary human hepatocytes should be the system of choice for the evaluation of liverspecific functions in humans $(16,17)$. Since human hepatocytes are only available in limited numbers, the development of culture systems that allow the cultivation of differentiated and functional hepatocytes is of great importance. HEPAC ${ }^{2}$ constitutes a standardized and validated long-term, serum-free human hepatocyte culture system in which hepatocyte morphology and function can be maintained for several weeks (18). In the present study, CHP was tested in the HEPAC ${ }^{2}$ system and the results were compared with a preclinical toxicity study in rats and a clinical study in patients with urogenital tumors.

\section{Materials and methods}

Biochemical and immunological assays. LDH activities and urea were detected with a lactate dehydrogenase assay (Abbott, Wiesbaden, Germany; 7D69-20) and urea detection kit (7D75-20/-30) using Abbott's Architect ${ }^{\circledR}$ c8000 system following the manufacturer's protocols. Albumin release into the culture medium was detected by a sandwich ELISA for human albumin using antibodies and a reference serum from Bethyl (Natutec, Frankfurt, Germany). Affinity purified goat anti-human albumin (Bethyl A80-129A) was used as coating antibody, and goat anti-human albumin horseradish peroxidase conjugate (Bethyl A80-129P) for detection. Human reference serum (Bethyl RS10-110) served as a standard. The ELISA was performed at room temperature. CHP was quantitated in plasma and urine using a sensitive liquid-chromatography/ mass spectroscopy method.

Isolation and culture of human hepatocytes. Liver samples were obtained from three donors in accordance with an ethical survey of the ethics commission of the Aerztekammer Mecklenburg-Vorpommern. All donors were males (age range 40-74 years) with diagnoses of pulmonary disease, anoxia, adenocarcinoma and hepatocellular carcinoma, respectively.
Human hepatocytes were isolated from resections by threestep collagenase perfusion $(19,20)$. The viability of cells was determined by the trypan blue exclusion test. Only hepatocyte preparations with $>70 \%$ viability were used in the experiments described below. Cells were plated onto collagen-coated 6-well plates at a density of $10^{6}$ cells/well using MEM (Minimal Essential Medium containing $500 \mathrm{ng} / \mathrm{ml}$ insulin and $500 \mu \mathrm{g} / \mathrm{ml}$ gentamycin) and cultured under tissue culture conditions. The medium was changed after 3-12 h to Human Hepatocyte Maintenance Medium (11) containing $10 \mathrm{ng} / \mathrm{ml} \mathrm{HGF}$ and $20 \mathrm{ng} / \mathrm{ml}$ EGF. Thereafter, the medium was changed daily. Harvested culture medium was stored in aliquots at $4^{\circ} \mathrm{C}$ and subsequently assayed for cellular vitality (LDH release) and hepatocellular function (release of urea and albumin). LDH-, urea- and albumin-release served as quality control parameters to ensure the vitality and functionality of the hepatocytes (18). For the evaluation of the toxicity of CHP, human hepatocytes were isolated, incubated for four days in tissue culture and then supplemented with CHP $(0.32-32,000 \mu \mathrm{g} / \mathrm{ml})$ or vehicle for control purposes for $24 \mathrm{~h}$. Following exposure, the medium was replaced by CHP-free culture medium and the hepatocytes were cultured for a further $72 \mathrm{~h}$ prior to the assessment of the biochemical parameters.

Preclinical animal toxicity testing of CHP. Assessment of hepatotoxicity was performed as part of a repeated dose oral toxicity study of CHP in rats (28-day study). Three groups of rats were treated with the drug in graduated doses: $285 \mathrm{mg} / \mathrm{kg}$ $(\mathrm{n}=6), 507 \mathrm{mg} / \mathrm{kg}(\mathrm{n}=6)$ and $903 \mathrm{mg} / \mathrm{kg}(\mathrm{n}=12)$. One group was treated with the vehicle $(n=12)$. The following investigations were performed: body weight development, food consumption, laboratory diagnostics (hematology, clinical chemistry and urinalysis) and determination of organ weights at the end of the test period. Pathological and histopathological investigations were conducted as well.

Clinical Phase II study of CHP in patients with urogenital tumors. This study was performed by R. Eckert (Molecular and Clinical Research, Lebach, Germany). The patients received CHP orally four days/week for 3 weeks at $8 \mathrm{~g} /$ day. Tumor entities included renal pelvis cancer $(n=2)$, superficial bladder cancer $(n=4)$, advanced bladder cancer $(n=11)$ and advanced prostate cancer $(n=13)$. In this open label clinical Phase II study to evaluate safety and efficacy of CHP in urogenital cancer patients, the following parameters for liver enzymes were measured: ALAT, ASAT, $\gamma$-GT, AP, total plasma protein and bilirubin at different time points during the study: i) prior to the start of treatment, ii) day 1 - start of treatment, iii) day 8 - 7 days after the first administration of CHP, iv) day 15 - 14 days after the first administration of CHP, v) day $22-21$ days after the first administration of CHP, vi) day $30-29$ days after the first administration of CHP and 7 days after the last dose of CHP, vii) day 60 - 59 days after the first administration of CHP and $\sim 5$ weeks after the last dose, viii) day 90 or longer after the first administration of CHP and $\sim 9$ weeks after the last dose.

Statistics. Analysis of variance was performed using the Dunnett's test, comparing group means against one reference group, and the Student's t-test. 


\section{Results}

Effects of CHP on long-term cultures of human hepatocytes. In order to compare the effect of CHP on LDH-release, the human hepatocyte cultures of the three different donors were exposed to increasing concentrations of the drug for $24 \mathrm{~h}$ following the preculturing of hepatocytes for 3 days. LDH activities in the absence of CHP on days 4-5 were set equal to $100 \%$. LDH activities detected in the presence of CHP were then calculated relative to those in untreated control cells (Fig. 1A). Statistical analysis revealed that CHP in concentrations of $0.32-32,000 \mu \mathrm{g} / \mathrm{ml}$ did not alter LDH release. For the comparison of albumin production in all donors, concentrations measured in the absence of CHP on days 4-5 were set equal to $100 \%$. Albumin release in CHP-treated hepatocytes was then calculated relative to that found in untreated controls (Fig. 1B). CHP at concentrations between 0.32 and $320 \mu \mathrm{g} / \mathrm{ml}$ had no effect on the release of albumin in culture medium. High concentrations of $\mathrm{CHP}(3,200$ and $32,000 \mu \mathrm{g} / \mathrm{ml})$ led to a significant reduction in hepatocellular albumin production $(\mathrm{p}<0.05)$. For the comparison of urea release in all donors, urea production in the absence of CHP at days 4-5 was set equal to $100 \%$. Urea release in CHP-treated hepatocytes was then calculated relative to that found in untreated controls. CHP at concentrations of $0.32-320 \mu \mathrm{g} / \mathrm{ml}$ had no effect on the release of urea in culture medium (Fig. 1C). High concentrations of CHP $(3,200$ and $32,000 \mu \mathrm{g} / \mathrm{ml})$ led to a significant reduction in hepatocellular urea production $(\mathrm{p}<0.05$ and $<0.001$, respectively).

Preclinical toxicology testing in rats. The following relevant changes were observed in the parameters analyzed in the repeated dose oral toxicity study of CHP in rats (28-day study): for ALAT and ASAT, significant increases in the mean values were seen on day 29 in comparison to the vehicle group after administration of $903 \mathrm{mg} / \mathrm{kg}$ test substance (Fig. 2). Albumin was increased in animals that received $507 \mathrm{mg} / \mathrm{kg}$ $\mathrm{CHP}$, and blood urea nitrogen was increased in animals that received $903 \mathrm{mg} / \mathrm{kg}$ CHP. For AP, a reduction in the mean values was seen on day 29 in comparison to the vehicle group after administration of $903 \mathrm{mg} / \mathrm{kg} \mathrm{b} . \mathrm{w}$. of test substance. However, the value was within the physiological range based on reference data of the species and strain (data not shown). Pathological inspection revealed a pale appearance of the liver due to drug-induced anemia in the highest dose level applied. Apart from this finding, no other abnormalities were observed.

Clinical application of CHP in patients with urogenital cancer. Results of the preparative in vitro and animal models were compared to one final human study regarding the alteration of liver enzymes during human exposition. In an open label clinical Phase II study to evaluate the safety and efficacy of CHP in urogenital cancer patients, the following parameters for liver enzymes were documented: liver parameters ALAT, ASAT and $\gamma$-GT were not elevated in this group of urogenital cancer patients under treatment with CHP (8 g/day, 4 days/ week for 3 weeks; Fig. 3). AP was reduced during the course of treatment, possibly due to reduced release from bone metastases in advanced prostate cancer patients. Total plasma protein and bilirubin exhibited no significant changes under therapy with CHP (data not shown).
A

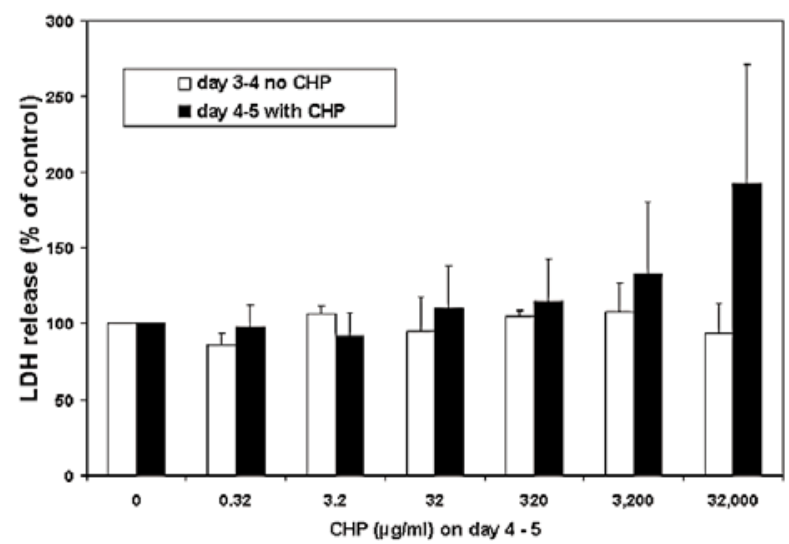

B

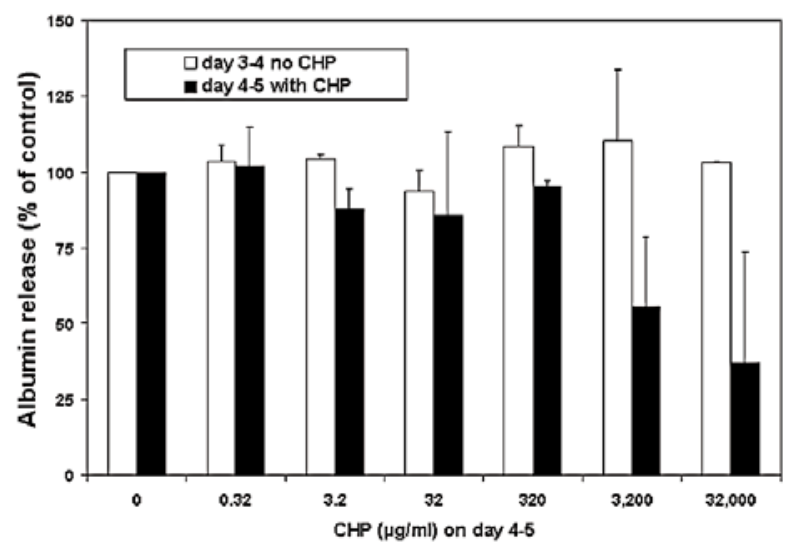

C

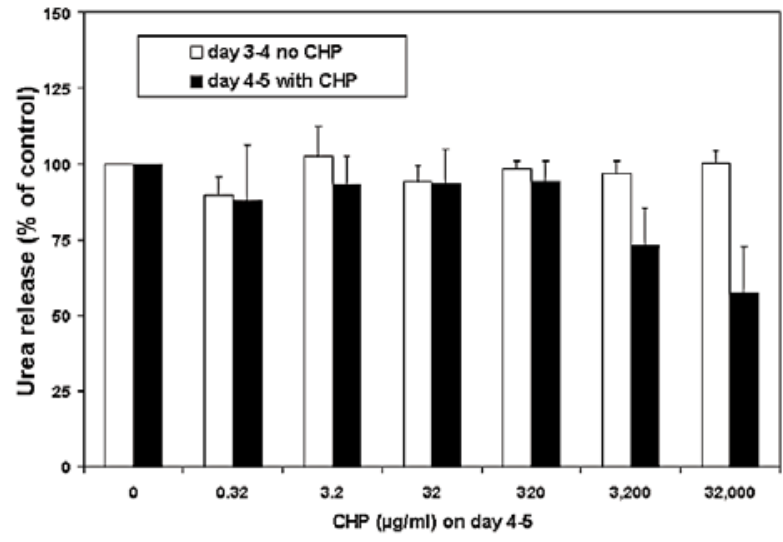

Figure 1. Determinations of LDH, albumin and urea release in cultures of human hepatocytes. Following isolation, cells were cultured for three days and the tissue culture medium from the last $24 \mathrm{~h}$ of incubation was used to measure the control values of all parameters (set to $100 \%$ ). For the next $24 \mathrm{~h}$, hepatocytes were exposed to the indicated concentrations of CHP and, after three days of a second culture period, function parameters were measured again and compared to respective controls. Data are presented as mean \pm SD $(\mathrm{n}=3)$. All differences for $\mathrm{CHP}=32,000 \mu \mathrm{g} / \mathrm{ml}$ are significant, as are those of albumin and $3,200 \mu \mathrm{g} / \mathrm{ml} \mathrm{CHP}$.

\section{Discussion}

The extracellular matrix, composed of collagens, proteoglycans, glycoproteins, elastin and other components, plays a key role in tumor angiogenesis, proliferation and dissemination. Collagens constitute a class of L-proline-rich proteins, and therefore analogs of L-proline such as CHP that affect collagens inhibit the growth of tumor cells in tissue culture and in vivo $(5,7,8)$. The incorporation of this amino acid analog into procollagen blocks the normal post-translational hydroxylation of L-proline residues, which are essential for the 

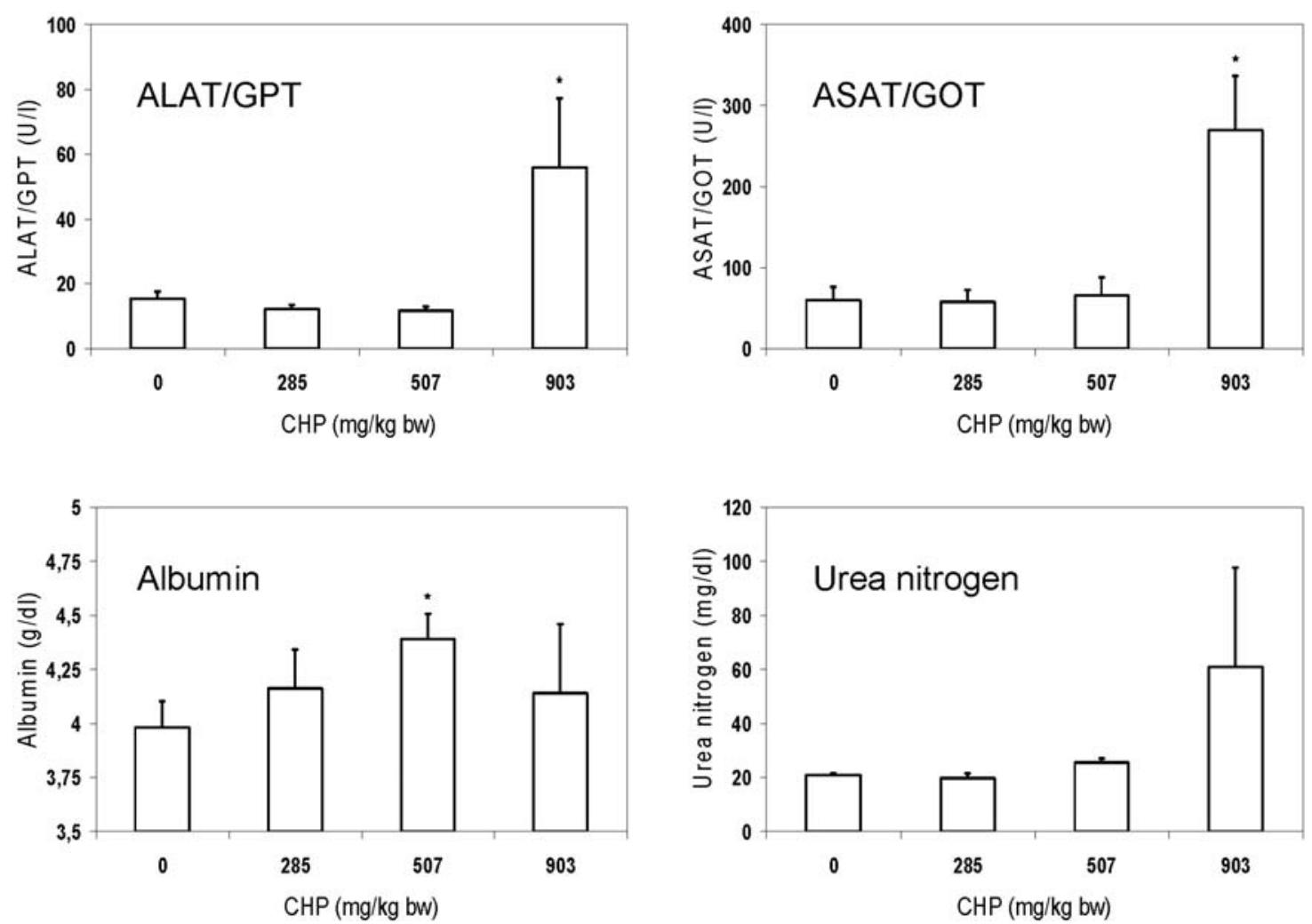

Figure 2. Liver function parameters measured on day 29 in the plasma samples of rats, treated for 28 days continuously with the indicated doses of CHP (mean \pm SD). ALAT, ASAT and urea nitrogen were significantly elevated in response to the highest dose of CHP ( $903 \mathrm{mg} / \mathrm{kg}$ ). Albumin was significantly elevated at a dose of $507 \mathrm{mg} / \mathrm{kg}$ CHP compared to untreated control animals.

stabilization of the triple helical configuration. The resulting non-helical collagen is ultimately degraded intracellularly rather than being deposited in the extracellular matrix (4). In addition, the cell death of tumor cells was found to be induced by endoplasmatic reticulum stress and cellular vacuolization $(9,10)$. Since incorporation of CHP may affect the function of non-collagenous proteins as well, hepatotoxicity has been a major concern during preclinical testing of this anticancer drug.

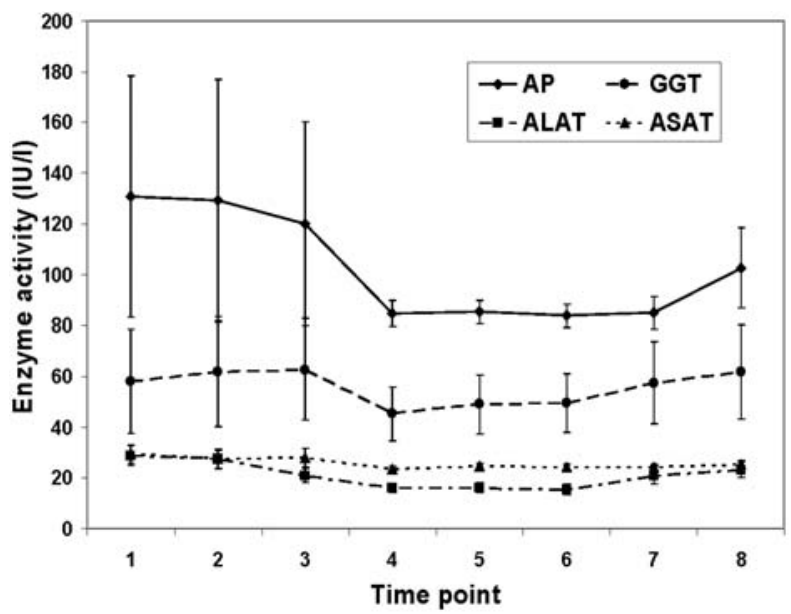

Figure 3. Course of liver function parameters ALAT, ASAT, $\gamma$-GT and AP in a group of 30 patients with urogenital tumors treated with CHP in a Phase II study (mean $\pm \mathrm{SD})$. Description of the time points can be found under Materials and methods. Time point 1 represents the pre-dosing values, and washout of CHP is observed starting from time point 6 .
Lee et al cultured isolated rat primary hepatocytes between two layers of collagen in a sandwich configuration that reinstates the cellular polarity necessary for long-term function in vitro (15). Maintenance of hepatocyte function, as measured by the secretion of albumin, was shown to be dependent on both the sandwich gel configuration and on the continued presence of L-proline in the culture media. CHP inhibited the response of sandwiched hepatocytes to L-proline in a dose-dependent and reversible manner. The addition of CHP to cultures established for seven days also resulted in an inhibition of hepatocyte function (15). Previously, insulin combined with epidermal growth factor (EGF) was demonstrated to markedly induce hepatocyte proliferation in L-proline-rich media (21). CHP strongly inhibited DNA synthesis without affecting protein production in the cells and without showing any cytotoxicity. These observations indicate that L-proline is essential for the induction of hepatocyte proliferation in culture, possibly through its effect on the synthesis of intracellular collagen. Provided that these findings are valid for human hepatocytes, CHP is expected to have adverse effects on liver function. Currently, preclinical analyses of new drugs require oral toxicity studies in rodents and non-rodents to evaluate the toxic characteristics of the drugs. Studies with rodents have to be designed according to OECD guidelines 407 (Repeated Dose 28-day Oral Toxicity Study in Rodents) and 408 (Repeated Dose 90-day Oral Toxicity Study in Rodents). Up to 60 animals are mandatory for each study. Often, the results obtained with these animal studies are not in line with data obtained from human studies (22). 
The aim of the present study was to investigate the effects of CHP on isolated human hepatocytes and to compare these data with results from preclinical animal testing and liver function parameters obtained in a Phase II clinical study in urogenital cancer patients. HEPAC ${ }^{2}$ is a validated serum-free long-term culture system for human hepatocytes developed by PRIMACYT (Schwerin, Germany) (18). During the process of establishment, several different parameters were used to evaluate hepatocellular integrity and hepatocellular function. To initially assess cellular vitality, several different parameters were included: releases of LDH, ALAT, ASAT and microscopic control. While ALAT activity was detectable in the culture medium within the first 6 days only, and was thereafter lost, ASAT and LDH release correlated and were detectable for several weeks. Therefore, in addition to microscopic control, LDH release was selected to monitor the viability of the cells. Albumin and urea are both synthesized and secreted by hepatocytes. Albumin can be used as a marker for the anabolic processes of hepatocytes and urea nitrogen as a marker for catabolism. Using the HEPAC ${ }^{2}$ system, significant adverse effects of CHP on human hepatocytes from three donors as assessed by $\mathrm{LDH}$ and urea were detected at concentrations exceeding 3,200 $\mu \mathrm{g} / \mathrm{ml}$. This level of CHP surpasses the clinical peak plasma concentration more than 10-fold; these data therefore predict the absence of CHP hepatoxicity in clinically relevant concentrations. The findings of Lee et al, describing an inhibitory effect of $120 \mu \mathrm{g} / \mathrm{ml} \mathrm{CHP} \mathrm{on} \mathrm{albumin}$ production in isolated rat hepatocytes, could not be confirmed using these isolated human hepatocytes with the HEPAC ${ }^{2}$ system (15).

In preclinical tests using rats, CHP was administered in doses of up to $903 \mathrm{mg} / \mathrm{kg}$ corresponding to plasma concentrations of up to $270 \mu \mathrm{g} / \mathrm{ml} \mathrm{CHP}(285 \mathrm{mg} / \mathrm{kg}$ to $60 \mu \mathrm{g} / \mathrm{ml}$, and $507 \mathrm{mg} / \mathrm{kg}$ to $180 \mu \mathrm{g} / \mathrm{ml} \mathrm{CHP).} \mathrm{Concentrations} \mathrm{of} \mathrm{ALAT}$ and ASAT were significantly elevated at the highest dose of CHP and albumin was increased at $507 \mathrm{mg} / \mathrm{kg}$, whereas urea tended to be increased at the highest dosage (not significant). In conclusion, preclinical testing in a rat model predicted an increased release of liver enzymes from hepatocytes near the clinical peak plasma concentrations of $\mathrm{CHP}$, pointing to possible adverse effects of this drug on liver function.

Clinical results for CHP were gathered in an open label Phase II study in 28 urogenital cancer patients, including local and advanced bladder cancer, prostate cancer and two cases of renal pelvic cancer. CHP was administered 4 days a week for 3 weeks ( $8 \mathrm{~g} /$ day). Peak plasma concentrations of CHP measured in a Phase I study using similar doses were approximately $200 \mu \mathrm{g} / \mathrm{ml}$. ALAT, ASAT, $\gamma$-GT and AP were determined in plasma samples gathered at regular intervals for 90 days. Elevations of ALAT and ASAT in the circulation correlated with the extent and severity of cellular liver damage. However, the differential diagnosis of liver disease requires the determination of $\gamma$-GT and AP in addition to ASAT, which is not specific to the liver. Levels of AP rise if there is a bile duct obstruction, intrahepatic cholestasis or infiltrative diseases of the liver. $\gamma$-GT is reasonably specific to the liver and is a sensitive marker that may be elevated with even minor subclinical levels of liver function. Data from the clinical observations of the CHP study indicate that no liver parameters were adversely affected, ASAT, ALAT, $\gamma$-GT and AP were not significantly different from pre-dosing values, and were within the normal reference range. In conclusion, the clinical data observed in this 28-patient group do not reveal signs of hepatoxicity for CHP.

Comparison of preclinical testing using $\mathrm{HEPAC}^{2}$ or rats with the clinical results indicates that $\mathrm{HEPAC}^{2}$ predicts the absence of toxicity correctly, whereas the experimental animals and the literature would have suggested the possibility of limited hepatotoxicity in humans $(15,21)$. Given the stability of the culture system regarding the maintenance of hepatocyte specific function and cytochrome P450 expression, HEPAC ${ }^{2}$ may also become an alternative to animal experiments for the assessment of the long-term effects of drugs and other chemicals. HEPAC ${ }^{2}$ allows differentiated human hepatocytes to be cultured for 28 days or longer. This should provide a tool for the analysis of acute or repeated dose effects that chemicals may exert on human hepatocytes. If other human cells could be maintained in a comparable fashion, it might be possible to reduce the number of animal studies required for the evaluation of the repeated dose effects of drugs.

The possibility exists that the HEPAC ${ }^{2}$ system could be used as a fast test system after new compounds have been synthesized to initially check for cellular toxicity; if any liver toxicity results then the development process can be reverted back to the compound design stage. This would reduce the numbers of animals needed in future studies, and make the entire process more cost-efficient. Furthermore, using the HEPAC $^{2}$ method to culture human hepatocytes could act as an early indicator, similar to a dose finding study.

\section{References}

1. Sistare FD and De George JJ: Preclinical predictors of clinical safety: opportunities for improvement. Clin Pharmacol Ther 82: 210-214, 2007.

2. Rodriguez-Frias EA and Lee WM: Cancer chemotherapy I: hepatocellular injury. Clin Liver Dis 11: 641-662, 2007.

3. Floyd J, Mirza I, Sachs B and Perry MC: Hepatotoxicity of chemotherapy. Semin Oncol 33: 50-67, 2006.

4. Takeuchi T and Prockop DJ: Biosynthesis of abnormal collagens with amino acid analogues. I. Incorporation of L-azetidine-2carboxylic acid and cis-4-fluoro-L-proline into protocollagen and collagen. Biochim Biophys Acta 175: 142-155, 1969.

5. Rosenboom J and Prockop DJ: Incorporation of cis-hydroxyL-proline into protocollagen and collagen. J Biol Chem 246: 1549-1555, 1971.

6. Uitto J, Hoffmann HP and Prockop DJ: Retention of non-helical procollagen containing cis-hydroxy-L-proline in rough endoplasmic reticulum. Science 190: 1202-1204, 1975.

7. Lewko WM, Liotta LA and Wicha MS: Sensitivity of Nnitrosomethylurea-induced rat mammary tumors to cis-hydroxyL-proline, an inhibitor of collagen production. Cancer Res 41: 2855-2862, 1981

8. Klohs WD, Steinkampf RW, Wicha MS, Mertus AE, Tunac JB and Leopold WR: Collagen-production inhibitors evaluated as antitumor agents. J Natl Cancer Inst 75: 353-359, 1985.

9. Mueller C, Emmrich J, Jaster R, Braun D, Liebe S and Sparmann G: Cis-4-hydrox-L-proline-induced inhibition of pancreatic cancer cell growth is mediated by endoplasmic reticulum stress. World J Gastroenterol 12: 1569-1576, 2006.

10. Olszewski U, Ellinger A, Zeillinger R, Baumgartner G and Hamilton G: Involvement of 14-3-3sigma in cytotoxicity of cis4-hydroxy-L-proline (CHP) against epithelial tumor cell lines. FEBS 2008, Proceedings, C2-152.

11. Runge D, Michalopoulos GK, Strom SC and Runge DM: Recent advances in human hepatocyte culture. Biochem Biophys Res Commun 274: 1-3, 2000.

12. Van de Bovenkamp M, Groothuis GM, Meijer DK and Olinga P: Liver fibrosis in vitro: cell culture models and precision-cut liver slices. Toxicol In Vitro 21: 545-557, 2007. 
13. Battle T and Stacey G: Cell culture models for hepatotoxicology. Cell Biol Toxicol 17: 287-299, 2001.

14. Nussler AK, et al: The suitability of hepatocyte culture models to study various aspects of drug metabolism. ALTEX 18: 91-101, 2001.

15. Lee J, Morgan JR, Tompkins RG and Yarmush ML: L-prolinemediated enhancement of hepatocyte function in a collagen gel sandwich culture configuration. FASEB J 7: 586-591, 1993.

16. Moshage $\mathrm{H}$ and Yap SH: Primary cultures of human hepatocytes: a unique system for studies in toxicology, virology, parasitology and liver pathophysiology in man. J Hepatol 3: 404-413, 1992.

17. Li AP: Human hepatocytes: isolation, cryopreservation and applications in drug development. Chem Biol Interact 168: 16-29, 2007.

18. Ullrich A, Berg C, Hengstler JG and Runge D: Use of a standardised and validated long-term human hepatocyte culture system for repetitive analyses of drugs: repeated administrations of acetaminophen reduces albumin and urea secretion. ALTEX 24: 35-40, 2007.

19. Strom SC, Jirtle RL, Jones RS, et al: Isolation, culture and transplantation of human hepatocytes. J Natl Cancer Inst 68: 771-778, 1982.
20. Strom SC, Monteith DL, Manoharan K and Novotny AL: Genetic toxicology studies with human hepatocytes. In: The Isolated Hepatocyte: Use in Toxicology and Xenobiotic Biotransformation. Rauckman EJ and Padilla GM (eds). Academic Press, Orlando, FL, pp265-280, 1987.

21. Nakamura T, Teramoto H, Tomita Y and Ichihara A: L-proline is an essential amino acid for hepatocyte growth in culture. Biochem Biophys Res Commun 122: 884-891, 1984.

22. Runge D, Köhler C, Kostrubsky VE, Jaeger D, Lehmann T, Runge DM, May U, Beer Stolz D, Strom SC, Fleig WE and Michalopoulos GK: Induction of cytochrome P450 CYP1A1, CYP1A2 and CYP3A4 but not of CYP2C9, CYP2C19, multidrug resistance (MDR-1) and multidrug resistance associated protein (MRP-1) by prototypical inducers in human hepatocytes. Biochem Biophys Res Commun 273: 333-341, 2000.

23. Li AP, Maurel P, Gomez-Lechon MJ, et al: Preclinical evaluation of drug-drug interaction potential: present status of the application of primary human hepatocytes in the evaluation of cytochrome P450 induction. Chem Biol Interact 107: 5-16, 1997. 